\title{
Impact of chronic wounds of various etiology on systemic profiles of key inflammatory cytokines, chemokines and growth factors, and their interplay
}

\author{
Małgorzata Krzystek-Korpacka ${ }^{1, A-F}$, Krzysztof Kędzior, ${ }^{1, C, E, F}$, Leszek Masłowski2,A,B,E,F, \\ Magdalena Mierzchałal, ${ }^{1,-F}$, Iwona Bednarz-Misa ${ }^{1, C, D, F}$, Agnieszka Bronowicka-Szydełk0 1,B,E,F, \\ Joanna Kubiak 2,B,E,F, Małgorzata Gacka ${ }^{3, B, E, F}$, Sylwia Płaczkowska ${ }^{4, C, E, F}$, Andrzej Gamian ${ }^{1, A, E, F}$ \\ ${ }^{1}$ Department of Medical Biochemistry, Faculty of Medicine, Wroclaw Medical University, Poland \\ 2 Department of Angiology, Regional Specialist Hospital, Wrocław, Poland \\ ${ }^{3}$ Department of Angiology, Hypertension and Diabetes, Wrocław Medical University, Poland \\ ${ }^{4}$ Department of Professional Training in Clinical Chemistry, Wroclaw Medical University, Poland \\ A - research concept and design; B - collection and/or assembly of data; $\mathrm{C}$ - data analysis and interpretation; \\ $D$ - writing the article; $E$ - critical revision of the article; $F$ - final approval of the article
}

Address for correspondence

Małgorzata Krzystek-Korpacka

E-mail:malgorzata.krzystek-korpacka@umed.wroc.pl

Funding sources

Statutory funding of Wroclaw Medical University \#ST-911.

Conflict of interest

None declared

\section{Acknowledgements}

The authors would like to thank the Foundation of Wroclaw Medical University (FAM) and its Board Chairmen for financing a lease of the BioPlex 200 platform.

Received on July 28, 2018

Reviewed on September 15, 2018

Accepted on February 8, 2019

Published online on August 19, 2019

\section{Cite as}

Krzystek-Korpacka M, Kędzior K, Masłowski L, et al. Impact of chronic wounds of various etiology on systemic profiles of key inflammatory cytokines, chemokines and growth factors, and their interplay. Adv Clin Exp Med. 2019:28(10):1301-1309. doi:10.17219/acem/103845

DOI

10.17219/acem/103845

Copyright

Copyright by Author(s)

This is an article distributed under the terms of the Creative Commons Attribution Non-Commercial License (http://creativecommons.org/licenses/by-nc-nd/4.0/)

\begin{abstract}
Background. Non-healing wounds are becoming a growing concern for public health as a result of their increasing prevalence in progressively aging societies.

Objectives. The aim of this article is to evaluate the effects of wound etiology on a panel of circulating cytokines in patients with non-healing wounds of the lower extremities.

Material and methods. This prospective case-control study involved 104 individuals: healthy elderly people $(n=46)$ and patients with diabetes and/or cardiovascular disease $(n=58$; among them 38 with chronic wounds of venous, ischemic or neurotrophic etiology). Selected serum cytokines - i.e. IL-1B, IL-4, IL-6, IL-8, FGF-2, G-CSF, GM-CSF, MCP-1, MIP-1a, TNF-a, VEGF-A, and PDGF-BB - were measured using the Luminex platform.
\end{abstract}

Results. Compared to healthy elderly people, presence of diabetes and/or cardiovascular disease was associated with elevated IL-6, IL-8, MCP-1 and G-CSF while non-healing wounds coexisted with the increase in the levels of all examined cytokines/growth factors except for G-CSF and GM-CSF. Among diseased elderly people, having wounds was associated with increased levels of IL-13, IL-4, IL-6, IL-8, FGF-2, MIP-1a, PDGF-BB, and VEGF-A. Interleukin $1 \beta$ elevation was a sole independent predictor of chronic wounds with an odds ratio $(O R)$ of 6.3. Cytokines in healthy seniors were loosely interrelated, while the levels of cytokines in diseased patients with wounds displayed a tight pattern of association. When stratified by their etiology, the association pattern for IL-6, IL-8, MCP-1, and VEGF-A was disrupted in neurotrophic wounds.

Conclusions. The results presented herein may improve our understanding of the pathomechanisms which lead to chronic wounds and of the effects they exert on a systemic level, as well as providing potential targets for more effective therapies.

Key words: diabetes, venous stasis, ischemic wounds, neurotrophic wounds, inflammation 


\section{Introduction}

Conditions such as diabetes or atherosclerosis can disturb blood flow, damage blood vessels, and, if severe, result in ulcerations or gangrene, most often located in the lower limbs. Moreover, together with age and obesity, they are listed as key factors which adversely affect proper wound healing. Taking into account the prevalence of obesity, diabetes and cardiovascular disease among elderly people, chronic wounds of the lower extremities are becoming a growing socioeconomic problem for aging societies. Persistent, frequent and often infected, they reduce quality of life, rendering afflicted persons disabled and in need of repeated hospitalization. ${ }^{1,2}$ Non-healing wounds are a major cause of morbidity and mortality and are responsible for over $80 \%$ of diabetes-associated amputations. ${ }^{3,4}$ Chronic wounds are currently estimated to affect up to $2 \%$ of the general population but - because their prevalence is increasing - it is predicted that they will affect $1 / 4$ of elderly people by the year $2050 .^{5}$

While the proper healing of an injury requires a sequence of events, tightly orchestrated in time and space by a plethora of humoral mediators, chronic wounds are believed to be locked in the initial inflammatory phase without resolution. ${ }^{4}$ A deregulated cytokine and growth factor network which promotes an inflammatory response but causes aberrations in immune cell recruitment, shifts in the proteolytic balance and impaired formation of blood vessels is implicated in the pathogenesis of chronic wounds. ${ }^{6}$ So far, most of the attention has been focused on profiling cytokines and growth factors in wound fluids and biopsies, as they are believed to reflect the microenvironment of non-healing wounds best, whereas the pathogenic significance of the accompanying systemic inflammation has received little attention. ${ }^{7}$ However, alterations in local cytokine and growth factor concentrations are unlikely to contribute to the systemic effects of chronic wounds, such as considerably higher mortality rates. ${ }^{8,9}$ Also, the collection and analysis of wound exudates pose some technical and interpretational problems..$^{10}$ Even more importantly, high proteolytic activity - a hallmark of non-healing wounds ${ }^{4}$ - is likely to falsify any results.

Thus, the purpose of our study was to profile, on a systemic level, the key pro-inflammatory and pro-angiogenic cytokines and growth factors and their interplay in healthy elderly people compared with seniors burdened with chronic conditions like diabetes and cardiovascular disease, either without or with complications in the form of chronic wounds. Also, we aimed to compare the cytokine profiles and correlation patterns in patients with chronic wounds, stratified by wound type and etiology.

\section{Material and methods}

\section{Study population}

The study population consisted of 104 individuals: 48 apparently healthy seniors and 56 elderly patients with diabetes and/or cardiovascular disease - 38 of whom had chronic wounds of the lower extremities. Patients with chronic wounds were recruited from the Department of Angiology of the Regional Specialist Hospital in Wrocław, Poland. Only patients with chronic wounds in the course of cardiovascular disease or diabetes were included, while patients with non-healing wounds due to autoimmune diseases, malignancy, infections, or drugs were excluded. Wound etiology was determined by its characteristics (location and an appearance of the wound, its borders, and the surrounding skin, pain, and the presence of bleeding on manipulation) in conjunction with the patient's history and clinical assessment based on the ankle-brachial pressure index, ultrasound, angiography, and computed tomography (CT), among other things. The wound etiology was determined to be as follows: venous stasis $(\mathrm{n}=17)$, ischemic (arterial) $(\mathrm{n}=13)$ and neurotrophic $(\mathrm{n}=6)$; in 2 cases, the dominant component was unclear (mixed ischemic/venous). Many of the patients $(n=27)$ exclusively had ulcerations, 5 had ulcerations and gangrene, and 6 had gangrene alone. Of the 11 patients with gangrene, 6 had wet gangrene and 5 had dry gangrene. Phlegmons were present in 5 patients. Data on hematological (hemoglobin, Hb; white blood cells, WBC; and platelets, PLT), coagulation (activated partial thromboplastin time, APTT) and biochemical (high-sensitive C-reactive protein, hsCRP; and fibrinogen) indices were prospectively collected and measured according to standard procedures.

Twenty age-matched patients with a similar chronic disease burden (type 2 diabetes associated with hypertension, hyperlipidemia, micro- and/or macroangiopathy, ischemic heart disease, or peripheral artery occlusive disease) but no limb ulcerations were recruited from the Department of Angiology, Hypertension, and Diabetes of the Wroclaw Medical University as a reference. Age and sex-matched individuals with complaints of headaches and memory loss but without mild cognitive impairment or dementia and no other significant health history recruited from the Research, Science, and Educational Center of Dementia Diseases in Ścinawa, Poland served as an additional control group. The age distribution in these 3 groups was as follows: $68.3 \pm 12.2$ years, $65.7 \pm 10.2$ years and $64.4 \pm 9.8$ years ( $\mathrm{p}=0.257$ ), respectively, while the female-to-male ratios were 17:21, 14:6 and 24:22 ( $\mathrm{p}=0.185)$, respectively.

The study conforms to the ethical principles outlined in the Declaration of Helsinki. The study design was approved by the Medical Ethics Committees of Wroclaw Medical University and the Regional Specialist Hospital, and informed consent was obtained from the patients. 


\section{Analytical methods}

Blood was drawn with venipuncture and was then clotted $\left(30^{\prime}\right)$ and centrifuged $(15,720 \times \mathrm{g})$. The resulting serum was frozen at $-80^{\circ} \mathrm{C}$ until examination. Cytokine profiling was conducted in duplicate with flow cytometry-based method using magnetic microspheres conjugated with monoclonal antibodies using a BioPlex 200 (Bio-Rad, Hercules, USA), according to the manufacturer's instructions, incorporating Luminex $\mathrm{xMAP}^{\circledR}$ technology and validated custom plexes allowing for simultaneous measurement of interleukin (IL)-1 $\beta$, IL-4, IL-6, IL-8, fibroblast growth factor (FGF)-2, granulocyte colony-stimulating factor (G-CSF), granulocyte-macrophage colony-stimulating factor (GM-CSF), monocyte chemoattractant protein (MCP)-1, macrophage inflammatory protein (MIP)- $1 \alpha$, tumor necrosis factor (TNF)- $\alpha$, vascular endothelial growth factor (VEGF)-A, and platelet-derived growth factor (PDGF)-BB. Standard curves were drawn using 4- or 5-parameter logistic (PL) regression and the data was analyzed using BioPlex Manager v. 6.0 software (BioRad).

\section{Statistical analysis}

The data distribution was tested with the KolmogorovSmirnov test and equality of variances was tested using Levene's test. Intergroup differences were analyzed using one-way analysis of variance (ANOVA) and the t-test for independent samples with Welch correction, where appropriate, or the Kruskal-Wallis H test or Mann-Whitney $\mathrm{U}$ test. The data is presented as means or medians with a $95 \%$ confidence interval $(95 \% \mathrm{CI})$. Frequency analysis was conducted using the $\chi^{2}$ test. Patterns of univariate correlations were established using the Pearson's or Spearman's tests. For limited data sets, the Spearman's test was applied, as it is less sensitive to outliers, and the results were additionally verified with the Kendall test. Logistic regression was conducted using the stepwise method with $\mathrm{p}=0.05$ and $\mathrm{p}=0.1$ as entrance and removal criteria. All calculated probabilities were two-tailed and $p$-values $\leq 0.05$ were considered statistically significant. The analyses were performed using MedCalc ${ }^{\circledR}$ v. 14.10.2 (MedCalc Software, Mariakerke, Belgium) statistical software.

\section{Results}

\section{Circulating cytokines in patients with chronic wounds and age-matched controls with or without comparable chronic disease burden}

We compared the levels of circulating cytokines in patients with chronic wounds with those found in individuals with a comparable disease burden (cardiovascular disease or diabetes) but without chronic wounds, and with those of elderly people without significant medical history, who served as age-matched controls. Interleukin 6, IL-8, G-CSF, and MCP-1 were the only cytokines which were significantly higher in the patients without wounds than in the controls. In turn, compared to the healthy controls, the patients with chronic wounds had elevated levels of all cytokines apart from G-CSF and GM-CSF. The elevation of IL-1 $\beta$, IL-4, IL-6, IL-8, FGF-2, MIP-1 $\alpha$, PDGF-BB, and VEGF-A was more accentuated in patients with chronic wounds than in patients with similar disease burden but no wounds (Table 1).

In a logistic regression analysis (stepwise method), an increase in IL-1 $\beta$ alone was an independent predictor

Table 1. Systemic levels of key inflammatory cytokines and growth factors in the study population

\begin{tabular}{|c|c|c|c|c|}
\hline Cytokine/growth factor & Patients with chronic wounds (W) & Diseased seniors (D) & Controls (C) & $\mathrm{p}$-value \\
\hline $\mid L-1 \beta[n g / L]$ & $6.5(5.4-8.3)^{\mathrm{D}, \mathrm{C}}$ & $0(0.0-0.4)^{\cup}$ & $0(0.0-0.0)^{W}$ & $<0.001^{\mathrm{K}}$ \\
\hline IL-4 [ng/L] & $5.9(4.7-7.7)^{\mathrm{D}, \mathrm{C}}$ & $1.5(1.2-1.9)^{\cup}$ & $1.3(1.2-1.6)^{W}$ & $<0.001^{\mathrm{K}}$ \\
\hline IL-6 [ng/L] & $23.3(18.5-34.6)^{\mathrm{D}, \mathrm{C}}$ & $6.9(5.3-10.1)^{\cup, C}$ & $4.6(3.7-5.0)^{W, D}$ & $<0.001^{\mathrm{K}}$ \\
\hline IL-8 [ng/L] & $139.6(97.6-173.7)^{\mathrm{D}, \mathrm{C}}$ & $16.6(11.4-24.1)^{\cup, C}$ & $10.7(9.2-13.5)^{W, D}$ & $<0.001^{\mathrm{K}}$ \\
\hline FGF2 [ng/L] & $32.1(28.1-34.4)^{\mathrm{D}, \mathrm{C}}$ & $4.3(0.2-6.3)^{\cup}$ & $4.1(2.7-11.3)^{W}$ & $<0.001^{\mathrm{K}}$ \\
\hline G-CSF [ng/L] & $24.3(21.3-27.7)$ & $32.7(25.4-43.1)^{c}$ & $21.2(17.8-25.3)^{\mathrm{D}}$ & $0.009^{A}$ \\
\hline GM-CSF [ng/L] & $13.8(11.0-18.4)$ & $11.3(7.0-22.7)$ & $15.2(11.7-19.4)$ & $0.961^{\mathrm{K}}$ \\
\hline MCP-1 [ng/L] & $70.2(59.6-82.7)^{C}$ & $66.8(53.4-83.6)^{c}$ & $38.5(30.9-48.0)^{W, D}$ & $<0.001^{\mathrm{A}}$ \\
\hline MIP-1a [ng/L] & $11.1(9.7-13.6)^{D, C}$ & $1.4(0.6-1.9)^{\cup}$ & $1.7(1.1-2.4)^{W}$ & $<0.001^{\mathrm{K}}$ \\
\hline PDGF-BB [ $\mu \mathrm{g} / \mathrm{L}]$ & $3.6(2.8-4.5)^{\mathrm{D}, \mathrm{C}}$ & $2.1(1.7-2.8)^{\mathrm{U}}$ & $1.72(1.4-2.1)^{\mathrm{W}}$ & $<0.001^{\mathrm{A}}$ \\
\hline $\mathrm{TNF}-\mathrm{a}[\mathrm{ng} / \mathrm{L}]$ & $14.6(11.0-18.6)^{c}$ & $12.9(9.4-15.3)$ & $10.6(7.8-14.1)^{\mathrm{w}}$ & $0.031^{\mathrm{K}}$ \\
\hline VEGF-A [ng/L] & $328(170.4-478.4)^{D, C}$ & $50.1(26.2-88.2)^{\cup}$ & $46.6(37.4-58.7)^{\mathrm{W}}$ & $<0.001^{\mathrm{K}}$ \\
\hline
\end{tabular}

Data is presented as means or medians with a 95\% confidence interval (95\% Cl) and was analyzed with one-way ANOVA or the Kruskal-Wallis $\mathrm{H}$ test. D - significantly different from diseased seniors without chronic wounds; W - significantly different from patients with chronic wounds; C - significantly different from apparently healthy age-matched controls; K - Kruskal-Wallis H test; A - one-way ANOVA; IL - interleukin; FGF2 - fibroblast growth factor 2; G-CSF - granulocyte colony-stimulating factor; GM-CSF - granulocyte-macrophage colony-stimulating factor; MCP-1 - monocyte chemoattractant protein-1; MIP-1a - macrophage inflammatory protein-1a; PDGF-BB - platelet-derived growth factor BB; TNF-a - tumor necrosis factor a; VEGF-A - vascular endothelial growth factor A. 
of the presence of chronic wounds $(b=1.84$, const. -5.26 ; $\mathrm{p}<0.0001)$ with an OR of $6.32(95 \% \mathrm{CI}=2.6-15.6)$, correctly classifying $99 \%$ of cases with an area under the curve $($ AUC) $=0.995(0.96-1.0)$.

\section{Correlations of circulating cytokines and growth factors with laboratory parameters in patients with chronic wounds}

Although there was no direct correlation with hemoglobin concentration, circulating IL-8 was significantly higher in the patients with hypochromia $(137.6 \mathrm{pg} / \mathrm{mL}$ (107-168) vs $212.5 \mathrm{pg} / \mathrm{mL}(163-263), \mathrm{p}=0.007)$ and MIP-1 $\alpha$ (12.2 pg/mL (10.2-14.3) vs 15.1 pg/mL (12.7-17.4), $\mathrm{p}=0.061)$, IL-6 (23.3 pg/mL (17.9-47.6) vs $42.2 \mathrm{pg} / \mathrm{mL}$ (26.5-133.2), $\mathrm{p}=0.071)$, and VEGF-A (332 pg/mL (133694) vs $614 \mathrm{pg} / \mathrm{mL}(326-1278), \mathrm{p}=0.087)$ tended to be higher as well. MCP-1 ( $\mathrm{r}=-0.5, \mathrm{p}=0.036)$ and PDGF-BB $(\mathrm{r}=-0.48, \mathrm{p}=0.042)$ were inversely correlated with activated partial thromboplastin time, an indicator of the efficacy of coagulation pathways, and a similar tendency was observed for IL-4 $(r=-0.46, p=0.056)$.

Apart from IL-6 $(\mathrm{r}=0.49, \mathrm{p}=0.009, \mathrm{n}=27)$, none of the studied cytokines and growth factors correlated with CRP. However, there were significant positive correlations with fibrinogen: TNF- $\alpha(r=0.52, p=0.012)$, IL-1 $\beta$ $(\mathrm{r}=0.48, \mathrm{p}=0.021), \mathrm{IL}-6(\mathrm{r}=0.45, \mathrm{p}=0.032), \mathrm{GM}-\mathrm{CSF}$ $(\mathrm{r}=0.47, \mathrm{p}=0.023), \mathrm{G}-\mathrm{CSF}(\mathrm{r}=0.46, \mathrm{p}=0.029)$, and FGF-2 $(\mathrm{r}=0.38, \mathrm{p}=0.073)$. The same held true for $\mathrm{WBC}$ count: TNF- $\alpha(r=0.57, p=0.002)$, MIP- $1 \alpha(r=0.42, p=0.031)$, IL-4 $(\mathrm{r}=0.46, \mathrm{p}=0.016), \mathrm{IL}-1 \beta(\mathrm{r}=0.54, \mathrm{p}=0.004)$, IL-6 $(\mathrm{r}=0.38, \mathrm{p}=0.050), \mathrm{IL}-8(\mathrm{r}=0.47, \mathrm{p}=0.014)$, GM-CSF $(\mathrm{r}=0.60, \mathrm{p}<0.001), \mathrm{G}-\mathrm{CSF}(\mathrm{r}=0.56, \mathrm{p}=0.002)$, and FGF-2 $(r=0.57, p=0.002)$. Likewise, positive correlations were
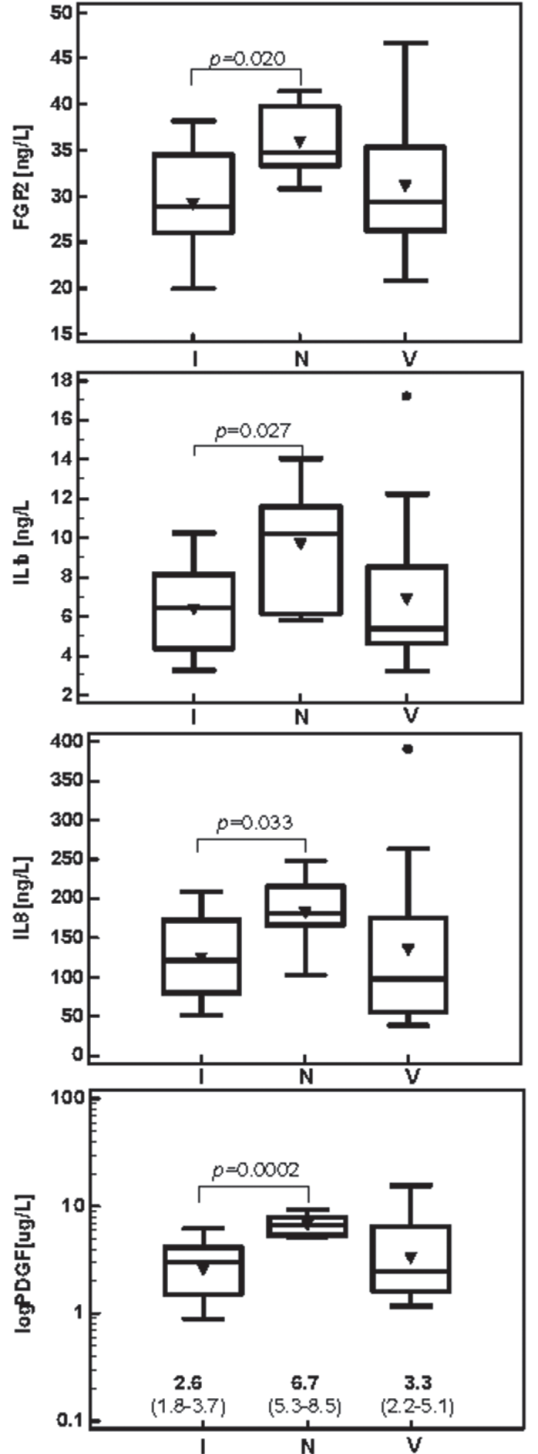
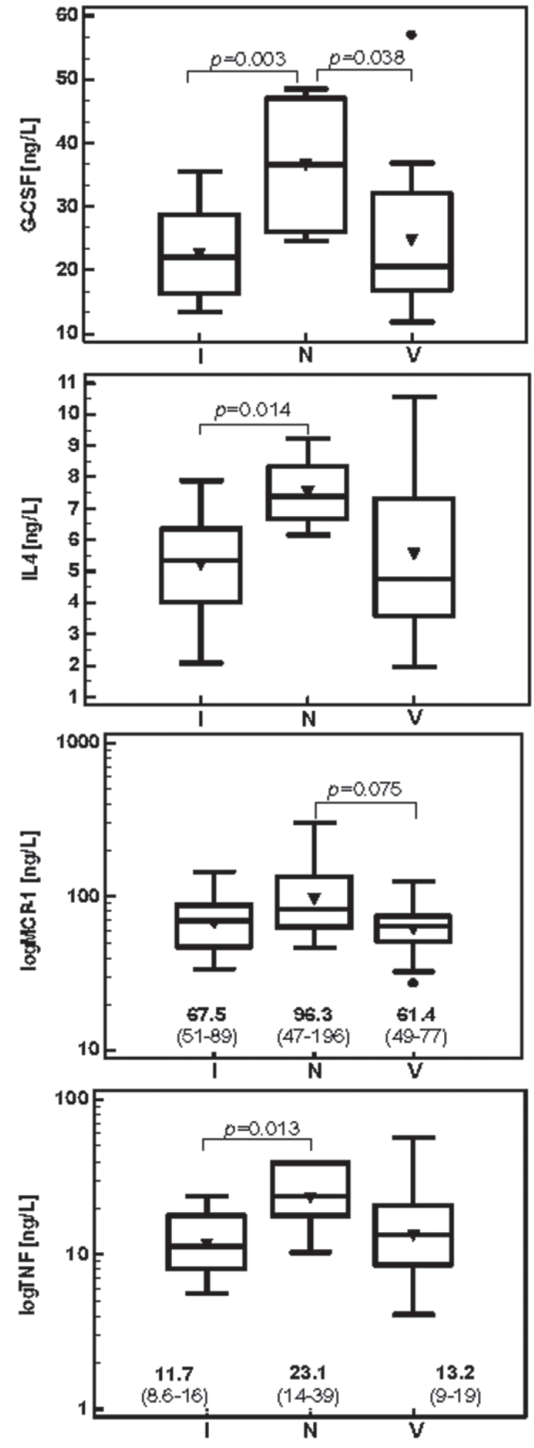
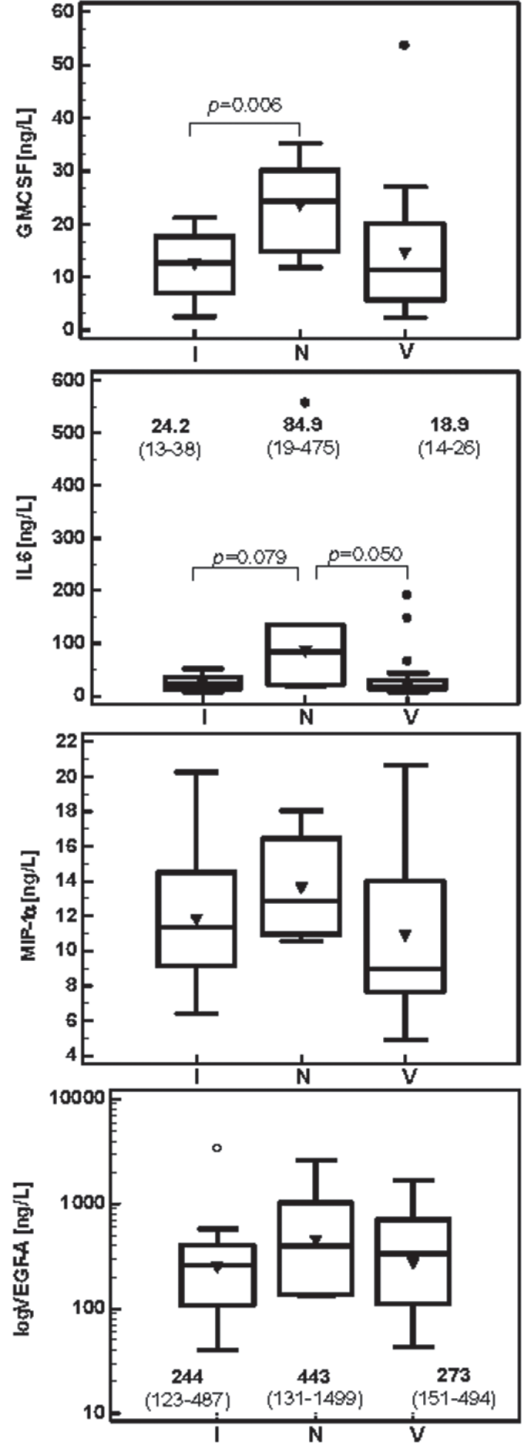

Fig. 1. Systemic levels of key inflammatory cytokines and growth factors in patients with chronic wounds stratified by wound etiology

I - patients with ischemic (arterial) wounds; $\mathrm{N}$ - patients with neurotrophic wounds; $\mathrm{V}$ - patients with vascular stasis wounds. Boxes represent interquartile range; bars inside boxes - medians; whiskers - 95\% confidence intervals ( $95 \% \mathrm{Cl}$ ); triangles - means. 
found between fibrinogen and PLT count: TNF- $\alpha(\mathrm{r}=0.44$, $\mathrm{p}=0.021), \operatorname{MIP}-1 \alpha(\mathrm{r}=0.52, \mathrm{p}=0.006), \mathrm{IL}-4(\mathrm{r}=0.38$, $\mathrm{p}=0.048), \mathrm{IL}-1 \beta(\mathrm{r}=0.43, \mathrm{p}=0.025), \mathrm{IL}-8(\mathrm{r}=0.53$, $\mathrm{p}=0.004)$, GM-CSF $(\mathrm{r}=0.45, \mathrm{p}=0.018)$, and G-CSF $(\mathrm{r}=0.39, \mathrm{p}=0.044)$.

\section{Correlations of circulating cytokines and growth factors with wound type}

There were tendencies towards more accentuated elevations of IL-6, IL-8, MIP-1 $\alpha$, and VEGF-A levels ( $\mathrm{p}=0.145$, $\mathrm{p}=0.186, \mathrm{p}=0.137$, and $\mathrm{p}=0.169$, respectively) in patients with both gangrene and ulcerations compared to those with ulcerations alone. There were no significant differences in circulating cytokines or growth factors in terms of the type of gangrene (wet vs dry) or the presence of phlegmon.

\section{Correlations of circulating cytokines and growth factors with wound etiology}

When stratified by wound etiology, there were significantly higher levels of IL-1 $\beta$, IL-4, IL8, FGF-2, G-CSF, GM-CSF, PDGF-BB, and TNF- $\alpha$ in patients with neurotrophic wounds than with ischemic ones. Interleukin 6 displayed a similar tendency. G-CSF and IL- 6 were significantly higher in neurotrophic wounds than venous ones as well (Fig. 1).

Moreover, G-CSF levels in patients with ischemic ( $p=0.015)$ and venous $(p=0.025)$ wounds were significantly lower than in individuals with a similar burden of chronic diseases but no wounds. While IL-1 $\beta$, IL-4, IL-6, IL-8, FGF-2, MIP-1 $\alpha$, and VEGF-A were significantly higher in patients with chronic wounds than in controls independent of their etiology, there were differences concerning PDGF-BB and TNF- $\alpha$. The wound-associated elevation of PDGF-BB in these patients compared to ones with a similar disease burden was significant exclusively in neurotrophic wounds ( $\mathrm{p}<0.001 ; \mathrm{p}=0.061$ for venous and $\mathrm{p}=0.384$ for ischemic). Compared to individuals without a significant medical history, there was no elevation of PDGF-BB in ischemic wounds ( $p=0.131$; $\mathrm{p}=0.0001$ for neurotrophic and $\mathrm{p}=0.003$ for venous). Tumor necrosis factor $\alpha$ was significantly higher in patients with neurotrophic wounds than in healthy controls $(\mathrm{p}=0.013 ; \mathrm{p}=0.179$ for ischemic and $\mathrm{p}=0.089$ for venous).

\section{Cytokine interplay}

The associations observed in the healthy elderly people were either nonexistent or less accentuated than those

Table 2. Cytokine correlation pattern in the study population

\begin{tabular}{|c|c|c|c|c|c|c|c|c|c|c|c|c|}
\hline $\begin{array}{c}\text { Cytokine/growth } \\
\text { factor }\end{array}$ & FGF-2 & G-CSF & GM-CSF & IL-1 $\beta$ & IL-4 & IL-6 & IL-8 & MCP-1 & MIP-1a & PDGF-BB & TNF-a & VEGF-A \\
\hline FGF-2 & - & $\begin{array}{c}\text { ns } \\
0.73^{\#}\end{array}$ & $\begin{array}{l}0.73^{\text {S\# }} \\
0.47^{*}\end{array}$ & $\begin{array}{c}\text { ns } \\
0.50 * 5\end{array}$ & $\begin{array}{l}0.51^{\text {S\# }} \\
0.75^{\#}\end{array}$ & $\begin{array}{c}\text { ns } \\
0.74^{\# S}\end{array}$ & $\begin{array}{c}\text { ns } \\
0.70^{\# S}\end{array}$ & $\begin{array}{c}-0.29^{5 *} \\
\text { ns }\end{array}$ & $\begin{array}{l}0.59^{\text {S\# }} \\
0.70^{\# S}\end{array}$ & $\begin{array}{l}0.63^{5 \#} \\
0.63^{+}\end{array}$ & $\begin{array}{l}0.39^{S+} \\
0.73^{\# S}\end{array}$ & $\begin{array}{c}\mathrm{ns} \\
0.60^{+}\end{array}$ \\
\hline G-CSF & $0.84^{\#}$ & - & $\begin{array}{l}\text { ns } \\
\text { ns }\end{array}$ & $\begin{array}{l}0.49^{S \#} \\
0.67^{\dagger S}\end{array}$ & $\begin{array}{l}0.52^{\#} \\
0.80^{\#}\end{array}$ & $\begin{array}{l}0.63^{5 \#} \\
0.81^{\# S}\end{array}$ & $\begin{array}{l}0.58^{\#} \\
0.76^{\# S}\end{array}$ & $\begin{array}{l}\text { ns } \\
\text { ns }\end{array}$ & $\begin{array}{l}0.32^{*} \\
0.75^{\# S}\end{array}$ & $\begin{array}{c}\mathrm{ns} \\
0.54^{*}\end{array}$ & $\begin{array}{l}0.60^{\#} \\
0.84^{\# S}\end{array}$ & $\begin{array}{l}0.59^{\#} \\
0.65^{\dagger}\end{array}$ \\
\hline GM-CSF & $0.89^{\#}$ & $0.92^{\#}$ & - & $\begin{array}{c}\text { ns } \\
0.48^{* 5}\end{array}$ & $\begin{array}{l}0.56^{\#} \\
0.47^{* 5}\end{array}$ & $\begin{array}{c}0.30^{5 *} \\
\mathrm{~ns}\end{array}$ & $\begin{array}{l}\text { ns } \\
\text { ns }\end{array}$ & $\begin{array}{c}-0.35^{\text {s* }} \\
\text { ns }\end{array}$ & $\begin{array}{l}\text { ns } \\
\text { ns }\end{array}$ & $\begin{array}{c}0.55^{\#} \\
\text { ns }\end{array}$ & $\begin{array}{l}0.39^{\dagger} \\
0.46^{* 5}\end{array}$ & $\begin{array}{l}\text { ns } \\
\text { ns }\end{array}$ \\
\hline IL-1 $\beta$ & $0.83^{\#}$ & $0.97^{\#}$ & $0.93^{\#}$ & - & $\begin{array}{l}0.39^{5+} \\
0.63^{5 t}\end{array}$ & $\begin{array}{l}0.58^{\text {S\# }} \\
0.62^{\text {St }}\end{array}$ & $\begin{array}{c}\text { ns } \\
0.51^{* s}\end{array}$ & $\begin{array}{l}\text { ns } \\
\text { ns }\end{array}$ & $\begin{array}{l}\text { ns } \\
\text { ns }\end{array}$ & $\begin{array}{c}\text { ns } \\
0.47^{* s}\end{array}$ & $\begin{array}{l}0.48^{5 \#} \\
0.73^{\# S}\end{array}$ & $\begin{array}{l}0.44^{S \dagger} \\
0.62^{\# S}\end{array}$ \\
\hline IL-4 & $0.86^{\#}$ & $0.91^{\#}$ & $0.82^{\#}$ & $0.89^{\#}$ & - & $\begin{array}{l}0.54^{\text {S\# }} \\
0.56^{\text {St }}\end{array}$ & $\begin{array}{c}\text { ns } \\
0.62^{\text {St }}\end{array}$ & $\begin{array}{l}\text { ns } \\
\text { ns }\end{array}$ & $\begin{array}{l}0.36^{*} \\
0.53^{* 5}\end{array}$ & $\begin{array}{l}0.51^{\#} \\
0.87^{\#}\end{array}$ & $\begin{array}{l}0.61^{\#} \\
0.70^{\# S}\end{array}$ & $\begin{array}{l}0.32^{*} \\
0.54^{*}\end{array}$ \\
\hline IL-6 & $0.42^{\mathrm{St}}$ & $0.68^{\text {S\# }}$ & $0.55^{\text {S\# }}$ & $0.62^{\text {S\# }}$ & $0.62^{\text {S\# }}$ & - & $\begin{array}{l}0.37^{5 *} \\
0.69^{\# S}\end{array}$ & $\begin{array}{l}\text { ns } \\
\text { ns }\end{array}$ & $\begin{array}{c}\text { ns } \\
0.54^{* s}\end{array}$ & $\begin{array}{l}\text { ns } \\
\text { ns }\end{array}$ & $\begin{array}{l}0.53^{\text {S\# }} \\
0.66^{\dagger S}\end{array}$ & $\begin{array}{l}0.59^{\text {S\# }} \\
0.74^{\# S}\end{array}$ \\
\hline IL-8 & $0.58^{\#}$ & $0.75^{\#}$ & $0.61^{\#}$ & $0.71^{\#}$ & $0.76^{\#}$ & $0.80^{\text {S\# }}$ & - & $\begin{array}{l}\text { ns } \\
\text { ns }\end{array}$ & $\begin{array}{c}\text { ns } \\
0.84^{\# S}\end{array}$ & $\begin{array}{l}\text { ns } \\
\text { ns }\end{array}$ & $\begin{array}{l}0.36^{*} \\
0.57^{+S}\end{array}$ & $\begin{array}{c}\text { ns } \\
0.61^{\dagger}\end{array}$ \\
\hline MCP-1 & $0.39 *$ & $0.49^{\dagger}$ & $0.50^{+}$ & $0.44^{\dagger}$ & $0.45^{\dagger}$ & $0.43^{S+}$ & $0.44^{\dagger}$ & - & $\begin{array}{l}\text { ns } \\
\text { ns }\end{array}$ & $\begin{array}{l}\text { ns } \\
\text { ns }\end{array}$ & $\begin{array}{l}\text { ns } \\
\text { ns }\end{array}$ & $\begin{array}{l}\text { ns } \\
\text { ns }\end{array}$ \\
\hline MIP-1a & $0.68^{\#}$ & $0.78^{\#}$ & $0.69^{\#}$ & $0.77^{\#}$ & $0.84^{\#}$ & $0.69^{\text {S\# }}$ & $0.75^{\#}$ & $0.40^{*}$ & - & $\begin{array}{c}0.37^{*} \\
\text { ns }\end{array}$ & $\begin{array}{c}0.53^{\#} \\
0.62^{\dagger S}\end{array}$ & $\begin{array}{c}\text { ns } \\
0.49^{* 5}\end{array}$ \\
\hline PDGF-BB & $0.88^{\#}$ & $0.86^{\#}$ & $0.89^{\#}$ & $0.86^{\#}$ & $0.88^{\#}$ & $0.55^{\mathrm{SH}}$ & $0.63^{\#}$ & $0.47^{\dagger}$ & $0.72^{\#}$ & - & $\begin{array}{c}0.31^{*} \\
0.52^{* 5}\end{array}$ & $\begin{array}{l}\text { ns } \\
\text { ns }\end{array}$ \\
\hline TNF-a & $0.76^{\#}$ & $0.85^{\#}$ & $0.85^{\#}$ & $0.86^{\#}$ & $0.75^{\#}$ & $0.55^{\text {S\# }}$ & $0.54^{\#}$ & $0.46^{\dagger}$ & $0.64^{\#}$ & $0.72^{\#}$ & - & $\begin{array}{c}0.51^{\#} \\
0.55^{* 5}\end{array}$ \\
\hline VEGF-A & $0.55^{\#}$ & $0.69^{\#}$ & $0.57^{\#}$ & $0.64^{\#}$ & $0.65^{\#}$ & $0.77^{\text {S\# }}$ & $0.77^{\#}$ & $0.46^{\dagger}$ & $0.72^{\#}$ & $0.53^{\#}$ & $0.51^{\dagger}$ & - \\
\hline
\end{tabular}

Unless otherwise indicated, data is presented as Pearson's correlation coefficients. ${ }^{*}-p<0.05 ;^{+}-p<0.01$; ${ }^{*}-p<0.001 ; S-S p e a r m a n ' s$ correlation coefficient; ns - not statistically significant. Correlations for the chronic wound group are presented on the left of the table. Correlations for the healthy elders are presented on the right of the table in regular font. Correlations for the diseased seniors without chronic wounds are presented on the right of the table in italic font. 
Table 3. Cytokine correlation pattern in patients with chronic wounds stratified by wound etiology

\begin{tabular}{|c|c|c|c|c|c|c|c|c|c|c|c|c|}
\hline $\begin{array}{l}\text { Cytokine/growth } \\
\text { factor }\end{array}$ & FGF-2 & G-CSF & GM-CSF & IL-1 $\beta$ & IL-4 & IL-6 & IL-8 & MCP-1 & MIP-1a & PDGF-BB & TNF- $a$ & VEGF-A \\
\hline FGF-2 & - & $\begin{array}{c}\text { ns } \\
0.69^{\dagger}\end{array}$ & $\begin{array}{l}0.94^{\dagger} \\
0.81^{\#}\end{array}$ & $\begin{array}{c}\text { ns } \\
0.71^{\dagger}\end{array}$ & $\begin{array}{l}0.83^{*} \\
0.75^{\dagger}\end{array}$ & $\begin{array}{l}\text { ns } \\
\text { ns }\end{array}$ & $\begin{array}{l}\text { ns } \\
\text { ns }\end{array}$ & $\begin{array}{l}\text { ns } \\
\text { ns }\end{array}$ & $\begin{array}{l}\text { ns } \\
\text { ns }\end{array}$ & $\begin{array}{l}1.00^{\#} \\
0.79^{\#}\end{array}$ & $\begin{array}{c}\text { ns } \\
0.65^{*}\end{array}$ & $\begin{array}{l}\text { ns } \\
\text { ns }\end{array}$ \\
\hline G-CSF & $0.78^{\#}$ & - & $\begin{array}{c}\text { ns } \\
0.88^{\#}\end{array}$ & $\begin{array}{l}0.94^{\dagger} \\
0.95^{\#}\end{array}$ & $\begin{array}{l}0.89^{*} \\
0.88^{\#}\end{array}$ & $\begin{array}{l}\text { ns } \\
\text { ns }\end{array}$ & $\begin{array}{c}\text { ns } \\
0.86^{\dagger}\end{array}$ & $\begin{array}{l}\text { ns } \\
\text { ns }\end{array}$ & $\begin{array}{l}0.94^{\dagger} \\
0.73^{\dagger}\end{array}$ & $\begin{array}{c}\text { ns } \\
0.56^{*}\end{array}$ & $\begin{array}{l}0.99^{\#} \\
0.81^{\#}\end{array}$ & $\begin{array}{l}\text { ns } \\
\text { ns }\end{array}$ \\
\hline GM-CSF & $0.87^{\#}$ & $0.84^{\#}$ & - & $\begin{array}{l}0.83^{*} \\
0.90^{\#}\end{array}$ & $\begin{array}{l}0.94^{\dagger} \\
0.80^{\#}\end{array}$ & $\begin{array}{l}\text { ns } \\
\text { ns }\end{array}$ & $\begin{array}{c}\text { ns } \\
0.67^{*}\end{array}$ & $\begin{array}{l}\text { ns } \\
\text { ns }\end{array}$ & $\begin{array}{l}0.83^{*} \\
0.67^{*}\end{array}$ & $\begin{array}{l}0.94^{\dagger} \\
0.62^{*}\end{array}$ & $\begin{array}{c}\text { ns } \\
0.89^{\#}\end{array}$ & $\begin{array}{l}\text { ns } \\
\text { ns }\end{array}$ \\
\hline $\mid L-1 \beta$ & $0.76^{\#}$ & $0.95^{\#}$ & $0.90^{\#}$ & - & $\begin{array}{l}0.94^{\dagger} \\
0.87^{\#}\end{array}$ & $\begin{array}{l}\text { ns } \\
\text { ns }\end{array}$ & $\begin{array}{c}\text { ns } \\
0.82^{\#}\end{array}$ & $\begin{array}{l}\text { ns } \\
\text { ns }\end{array}$ & $\begin{array}{l}1.00^{\#} \\
0.66^{*}\end{array}$ & $\begin{array}{c}\text { ns } \\
0.60^{*}\end{array}$ & $\begin{array}{l}0.93^{\dagger} \\
0.85^{\#}\end{array}$ & $\begin{array}{c}\text { ns } \\
0.56^{*}\end{array}$ \\
\hline $\mid \mathrm{L}-4$ & $0.76^{\#}$ & $0.96^{\#}$ & $0.76^{\#}$ & $0.92^{\#}$ & - & $\begin{array}{l}\text { ns } \\
\text { ns }\end{array}$ & $\begin{array}{c}\text { ns } \\
0.71^{\dagger}\end{array}$ & $\begin{array}{l}\text { ns } \\
\text { ns }\end{array}$ & $\begin{array}{l}0.94^{+} \\
0.78^{+}\end{array}$ & $\begin{array}{l}0.83^{*} \\
0.79^{+}\end{array}$ & $\begin{array}{l}0.87^{*} \\
0.75^{+}\end{array}$ & $\begin{array}{l}\text { ns } \\
\text { ns }\end{array}$ \\
\hline IL-6 & $0.53^{*}$ & $0.75^{\#}$ & $0.52^{*}$ & $0.64^{\dagger}$ & $0.66^{\dagger}$ & - & $\begin{array}{c}\text { ns } \\
0.63^{*}\end{array}$ & $\begin{array}{l}\text { ns } \\
\text { ns }\end{array}$ & $\begin{array}{l}\text { ns } \\
\text { ns }\end{array}$ & $\begin{array}{l}\text { ns } \\
\text { ns }\end{array}$ & $\begin{array}{l}\text { ns } \\
\text { ns }\end{array}$ & $\begin{array}{c}\text { ns } \\
0.80^{\#}\end{array}$ \\
\hline IL-8 & $0.60^{*}$ & $0.89^{\#}$ & $0.60^{*}$ & $0.79^{\#}$ & $0.86^{\#}$ & $0.92^{\#}$ & - & $\begin{array}{l}\text { ns } \\
\text { ns }\end{array}$ & $\begin{array}{c}\text { ns } \\
0.76^{\dagger}\end{array}$ & $\begin{array}{l}\text { ns } \\
\text { ns }\end{array}$ & $\begin{array}{c}\text { ns } \\
0.68^{*}\end{array}$ & $\begin{array}{c}\text { ns } \\
0.85^{\#}\end{array}$ \\
\hline MCP-1 & $0.49^{*}$ & $0.55^{*}$ & $0.51^{*}$ & $0.50^{*}$ & $0.48^{*}$ & $0.67^{\dagger}$ & $0.58^{*}$ & - & $\begin{array}{l}\text { ns } \\
\text { ns }\end{array}$ & $\begin{array}{l}\text { ns } \\
\text { ns }\end{array}$ & $\begin{array}{l}\text { ns } \\
\text { ns }\end{array}$ & $\begin{array}{l}\text { ns } \\
\text { ns }\end{array}$ \\
\hline MIP-1a & $0.71^{\#}$ & $0.92^{\#}$ & $0.73^{\#}$ & $0.91^{\#}$ & $0.94^{\#}$ & $0.69^{\dagger}$ & $0.84^{\#}$ & ns & - & $\begin{array}{c}\text { ns } \\
0.64^{*}\end{array}$ & $\begin{array}{l}0.93^{\#} \\
0.58^{*}\end{array}$ & $\begin{array}{c}\text { ns } \\
0.68^{+}\end{array}$ \\
\hline PDGF-BB & $0.87^{\#}$ & $0.94^{\#}$ & $0.86^{\#}$ & $0.92^{\#}$ & $0.94^{\#}$ & $0.63^{\dagger}$ & $0.80^{\#}$ & $0.49^{*}$ & $0.88^{\#}$ & - & $\begin{array}{l}\text { ns } \\
\text { ns }\end{array}$ & $\begin{array}{l}\text { ns } \\
\text { ns }\end{array}$ \\
\hline TNF-a & $0.69^{\dagger}$ & $0.80^{\#}$ & $0.88^{\#}$ & $0.90^{\#}$ & $0.76^{\#}$ & ns & $0.59 *$ & $0.55^{*}$ & $0.73^{\#}$ & $0.79^{\#}$ & - & $\begin{array}{l}\text { ns } \\
\text { ns }\end{array}$ \\
\hline VEGF-A & $0.65^{\dagger}$ & $0.81^{\#}$ & $0.57^{\dagger}$ & $0.69^{\dagger}$ & $0.81^{\#}$ & $0.85^{\#}$ & $0.88^{\#}$ & $0.64^{\dagger}$ & $0.81^{\#}$ & $0.71^{\#}$ & ns & - \\
\hline
\end{tabular}

${ }^{*}-<0.05 i^{\dagger}-<0.01 ;{ }^{*}-<0.001 ;$ ns - not statistically significant. Data is presented as Spearman's rank correlation coefficients. Correlations for venous etiology are presented on the left of the table. Correlations calculated for neurotrophic etiology (in regular font) and for ischemic etiology (in italic font) are presented on the right of the table.

in patients burdened with chronic diseases. Compared to patients with chronic wounds, there were no interrelationships for MCP-1 and only a few for IL-8, MIP-1 $\alpha$, PDGF-BB, and VEGF-A. In patients with chronic wounds, all cytokines were correlated and the statistical power of most of the observed associations was high (Table 2).

The analysis of patterns of cytokine correlation in patients with wounds stratified by wound etiology revealed a pattern of tight associations for venous wounds, while in ischemic wounds the associations were looser, even more so in neurotrophic wounds. The disruption of cytokine interrelationships in neurotrophic wounds was particularly evident for IL-6, IL-8, MCP-1, and VEGF-A - whose levels did not correlate with any other cytokine - and, to a lesser extent, for PDGF-BB and FGF-2 (Table 3).

\section{Discussion}

While there are number of studies which have profiled mediators of inflammation, angiogenesis and matrix remodeling in wound exudates, we focused on the levels of these mediators in circulation. The investigation of wound fluids is compelling, as it may potentially provide insight into what happens directly at the site of inflammation. However, due to the extremely high proteolytic activity which may falsely increase or decrease the availability of antigen epitopes for antibody-based assays or may cause artificial mass shifts in proteomic analyses, the examination of exudates may be equally misleading. It may account for reported increases in the levels of some cytokines with collection time and for a diminished ability of exudate to stimulate human dermal fibroblasts despite an elevated cytokine concentrations. ${ }^{11}$ Consequently, the results of such analyses are often contradictory, as exemplified by calcium-binding proteins S100A8 and $A 9$, the deficiency ${ }^{12}$ and overexpression ${ }^{13}$ of which have both been named a hallmark of non-healing wounds. Moreover, circulating mediators can both regulate local processes in a wound and exert an effect systemically. As such, profiling them may help to reveal the mechanisms responsible for systemic events contributing to the considerably higher mortality rates of diabetics with ulcerations than those without wounds $(\mathrm{R}=1.49)^{8}$ or of seniors with chronic wounds of various etiology compared to the age-matched general population (28 vs $4 \%)^{9}{ }^{9}$

Corroborating previous reports, ${ }^{7,14-17}$ all of our patients with diabetes and/or cardiovascular disease, regardless their wound status, had elevated levels of the cytokines characteristic of vascular inflammation and inflammatory milieu in diabetes: MCP-1, IL-6 and IL-8. MCP-1 is a key monocyte-attracting chemokine released from vascular endothelial cells (ECs) and smooth muscle cells (SMCs) during the initial phases of atherosclerosis. It facilitates trans-endothelial migration of adherent monocytes ${ }^{18}$ 
and induces EC and SMC migration as well as SMC proliferation. ${ }^{19}$

Circulating MCP-1 is believed to be a reliable marker of atherosclerotic plaque burden. ${ }^{20}$ As such, the equally high levels of MCP-1 in our diseased patients with and without chronic wounds confirms that both study groups were well-matched with respect to the degree of their affliction. Elevated MCP-1 in the serum of diabetic patients who developed foot ulcers has been predictive of healing failure. ${ }^{21}$

Interleukin 8 , primarily a neutrophil-attracting chemokine, is involved in the induction of monocyte adhesion to the endothelium, the stimulation of SMC proliferation and migration, and - in later stages - in the enhancement of plaque angiogenesis. ${ }^{18}$ Interleukin 6 , in turn, is one of the key leukocyte-derived pro-inflammatory cytokines which stimulates MCP-1 synthesis in macrophages and CRP expression in hepatocytes, as well as inducing SMC proliferation. ${ }^{22}$ However, inflammation is associated not only with the initiation of atherosclerosis, but also with its progression and the induction of plaque rupture. ${ }^{23} \mathrm{In}$ this respect, it is interesting that the elevation of IL-6 and IL-8 levels was more accentuated in diseased seniors with wounds than in those without wounds (3.4-fold and 8.4fold, respectively) and in diseased patients without wounds than in their healthy peers (1.5-fold). Interleukin 6 , as well as IL-6-induced expression of CRP and MCP-1 in plaque macrophages, causes an overexpression of tissue factor (TF) and hence activates the pro-coagulant pathway. Accordingly, there was an adverse correlation between MCP-1 and APTT which, if shortened, might be indicative of an increased risk of thromboembolism. Also, IL-6 enhances the expression of matrix metalloproteinases (MMPs), facilitating the disintegration of fibrous caps, and thus destabilizing plaques. ${ }^{19,22}$ Similarly, IL-8 induces the endothelial expression of MMP-2 and MMP- $-9^{24}$ and inhibits the expression of their inhibitor, TIMP- $1 .{ }^{25}$ As such, a more pronounced systemic elevation of these MMPs in combination with non-healing wounds promotes and sustains inflammatory milieu within blood vessels and may translate into the progression of atherosclerosis, plaque disruption and thrombosis. Accordingly, an elevation in circulating IL- ${ }^{18}$ or IL- 6 level ${ }^{16,19}$ is an independent predictor of cardiovascular events in various clinical settings. Locally, such a substantial upregulation of circulating IL-6 and IL-8 may contribute to enhanced proteolytic activity and a degradation of growth factors within wounds, further disturbing their proper healing.

In addition to IL- 6 and IL-8, IL-1 $\beta$ and MIP- $1 \alpha$ were also more markedly upregulated in patients with chronic wounds. Interleukin $1 \beta$ released from keratinocytes signals skin disruption, initiating and orchestrating the inflammatory response to injury. ${ }^{26}$ Accordingly, IL- $1 \beta$ alone was capable of correctly predicting the presence of wounds with $99 \%$ accuracy. Moreover, we observed a close correlation between IL-1 $\beta$ and all other evaluated cytokines and growth factors, which were either less accentuated or absent in individuals without chronic wounds. On the other hand, IL-1 $\beta$ is a pivotal activator of endothelial pro-coagulant activity and a suppressor of anticoagulant mechanisms, thereby contributing to microvascular thrombosis. ${ }^{22}$ Moreover, since lipid overload is one of the key factors contributing to plaque instability, ${ }^{23}$ the persistent upregulation of circulating IL-1 $\beta$, an inhibitor of cholesterol efflux regulatory protein (CERP), ${ }^{27}$ may facilitate atheroma rupture as well. Increased plaque infiltration with $\mathrm{T}$ cells and macrophages is yet another indicator of plaque instability. ${ }^{23}$ In this respect, only in patients with diabetes/cardiovascular disease and chronic wounds, an 8-fold upregulation of circulating MIP- $1 \alpha$ translates into accelerated migration of Th1 cells and cytotoxic CD8 $8^{+} \mathrm{T}$ lymphocytes into the inflamed vessels, ${ }^{28}$ the destabilization of existing atheromas, and - locally - the perpetuation and acceleration of inflammation within the wound. Indeed, an elevation in circulating MIP-1 $\alpha$ has been associated with short-term mortality in patients with acute coronary syndrome. ${ }^{29}$

Several studies have shown lower levels of growth factors within chronic wounds than acute ones. ${ }^{26}$ However, notwithstanding reservations concerning the reliability of wound examinations, it has been suggested that chronic wounds might not necessarily be deficient in growth factors but that the growth factors might be inefficient, being trapped within the fibrin cuffs surrounding the capillaries. ${ }^{30}$ Undoubtedly, the high activity of wound proteases is likely to degrade both endogenous and exogenous growth factors, rendering them ineffective as well. Moreover, the deregulation of downstream events has been demonstrated and is likely to contribute. ${ }^{26}$ Accordingly, in our patients with chronic wounds, the levels of key circulating growth factors necessary for proper wound healing were significantly higher: FGF-2 (7-fold), PDGF-BB (1.7-fold) and VEGF-A (8-fold). Corroborating our observations, an elevation in FGF-2 and PDGF-AA but not VEGF-A was associated with a failure for diabetic foot ulcers to heal. ${ }^{21}$ As with pro-inflammatory cytokines, the persistent systemic upregulation of growth factors might be detrimental. In fact, FGF-2, through the upregulation of MMP-2 and MMP-9 expression and activity, contributes to the thinning of the fibrous cap of an atheroma. ${ }^{31}$ VEGF-A has been shown to prompt apoptosis in macrophages, ${ }^{32}$ a phenomenon which is critical for resolving inflammation in normal wound healing but which contributes to atheroma rupture if it affects plaque macrophages. Moreover, VEGF-A induces tissue factor expression in the endothelium, whereas PDGF-BB is responsible for vascular smooth muscle cells, monocytes and macrophages. This, in turn, initiates clotting cascade and thrombus formation on the one hand, and the proliferation and migration of vascular SMCs leading to plaque progression, destabilization and rupture on the other hand. ${ }^{33}$

Apart from creating a pro-coagulant environment which promotes arterial and venous thrombosis, persistently elevated levels of pro-inflammatory cytokines may 
also contribute to the pathogenesis of anemia of chronic disease. Interleukin 6 plays a pivotal role in regulating levels of hepcidin, a major regulator of iron homeostasis. Additionally, pro-inflammatory cytokines directly affect erythropoiesis by inhibiting the synthesis of erythropoietin by or interfering with its signaling pathways. ${ }^{34}$ Accordingly, circulating IL-8 was significantly higher in patients with hypochromia, while IL-6, MIP-1 $\alpha$ and VEGF-A displayed a similar tendency.

Data concerning an association of IL-4 with wound healing are scarce. Animal models show the involvement of IL-4 in normal wound healing, where it activates fibroblasts and stimulates the synthesis of extracellular matrix. ${ }^{35}$ Interleukin 4 is involved in the activation of macrophages into a wound-healing phenotype as well. However, IL-4 has also been demonstrated to hamper the proangiogenic capacity of macrophages by downregulating hypoxia-inducible factor (HIF)- $1 \alpha$ translation. ${ }^{36}$ In vitro, stimulation with IL-4 reduced the migration of lung epithelial cells and hindered sinonasal epithelial wound closure ${ }^{37}$ Corroborating the notion that IL-4 contributes to prolonged healing, circulating IL-4 was significantly upregulated in patients with non-healing wounds. While other Th2 cytokines display an anti-atherogenic effect, the role of IL-4 remains ambiguous but there are suggestions that it is a promotor of plaque progression. ${ }^{27}$ Supporting this concept, IL-4 in our patients positively correlated with WBC and PLT counts and inversely with APTT.

Contrary to other cytokines, the systemic levels of G-CSF and GM-CSF were not elevated in our patients. In fact, G-CSF levels in patients with wounds of ischemic or venous etiology were significantly lower. G-CSF is a hematopoietic cytokine which plays a crucial role in the host response to infection. It is responsible for increasing the number of neutrophils in circulation by stimulating the proliferation, survival and differentiation of their precursors as well as their release from blood marrow. It is also believed to display immunomodulatory and antibiotic-enhancing activities; exogenous G-CSF application has been found to be beneficial as well. ${ }^{3}$ Locally, insufficient levels of G-CSF in patients with chronic wounds may contribute to the ineffectiveness of neutrophils infiltrating the wound in fighting infection. Decreased systemic G-CSF concentrations may render patients with infected chronic wounds more susceptible to severe complications in a form of bacterial infection of the surrounding skin and bones - or even sepsis, if the infection spreads to the circulatory system. GM-CSF promotes healing through many mechanisms, e.g., by increasing VEGF expression in the ulcer bed ${ }^{38}$ and an increased healing of chronic leg ulcers treated with GM-CSF has been shown. ${ }^{39}$ Beidler et al. ${ }^{40}$ demonstrated that higher systemic levels of GM-CSF at presentation were predictive of faster healing venous ulcers following multilayer compression therapy.

As pointed out by Trøstrup et al., ${ }^{11}$ the current knowledge on the differences or similarities between chronic wounds of various etiologies is insufficient, yet necessary to optimize treatment. To shed some light on the subject, we compared the profiles of circulating cytokines in patients stratified by wound etiology. Reflecting the prevalence in the general population, chronic wounds of venous etiology were the most common, whereas there were only a few cases of neurotrophic wounds in our study group. Nevertheless, neurotrophic wounds were associated with significantly higher levels of circulating IL-1 $\beta$, IL-4, IL-8, FGF-2, G-CSF, GM-CSF, PDGF-BB, and TNF- $\alpha$ than ischemic wounds and higher levels of IL- 6 and G-CSF than venous wounds.

Various cells involved in the tissue response to injury communicate through the cytokine network to orchestrate the event from inflammation induction to resolution and wound closure, making the proper interplay of cytokines critical. ${ }^{6}$ In addition to the differences in the systemic levels with respect to wound etiology, we observed a disruption of cytokine interrelationships in neurotrophic wounds. This was particularly evident for key inflammatory cytokines and chemokines - IL-6, IL-8 and MCP-1 - whose levels did not correlate with any other cytokine. The correlation pattern was also disrupted in the case of pro-angiogenic factors VEGF-A, PDGF-BB and FGF-2, supporting the notion that deregulation of their signaling and cross-talk plays a role in wound healing failure.

In conclusion, cytoprofiling revealed a pro-inflammatory state in patients with chronic wounds which might translate into enhanced pro-coagulant, pro-thrombotic and proteolytic activities that would locally contribute to prolonged healing or healing failure and - on a systemic level - may increase the risk of cardiovascular events and/or anemia of chronic disease. We also demonstrated that wound etiology affects the profile of circulating cytokines and growth factors as well as their interplay, altered particularly in patients with wounds of neurotrophic origin. Our findings may improve our understanding of the pathomechanisms leading to chronic wounds and the effects they exert on a systemic level, as well as providing potential targets for more effective therapies.

\section{ORCID iDs}

Małgorzata Krzystek-Korpacka (1) https://orcid.org/0000-0002-2753-8092 Krzysztof Kędzior (D) https://orcid.org/0000-0001-7206-8600 Leszek Masłowski: brak

Magdalena Mierzchała-Pasierb (1) https://orcid.org/0000-0003-0674-429X Iwona Bednarz-Misa (D) https://orcid.org/0000-0001-7244-2017 Agnieszka Bronowicka-Szydełko

(D) https://orcid.org/0000-0001-9967-036X Joanna Kubiak: brak

Małgorzata Gacka (D) https://orcid.org/0000-0001-5760-1534

Sylwia Płaczkowska (D) https://orcid.org/0000-0002-1466-3820 Andrzej Gamian (D) https://orcid.org/0000-0002-2206-6591

\section{References}

1. Rayner R, Carville K, Keaton J, Prentice J, Santamaria N. Leg ulcers: Atypical presentations and associated comorbidities. Wound Practice and Research. 2009;17:168-185. 
2. Green J, Jester R, McKinley R, Pooler A. The impact of chronic venous leg ulcers: A systematic review. J Wound Care. 2014;23(12):601-612.

3. de Lalla F, Pellizzer G, Strazzabosco M, et al. Randomized prospec tive controlled trial of recombinant granulocyte colony-stimulating factor as adjunctive therapy for limb-threatening diabetic foot infection. Antimicrob Agents Chemother. 2001;45(4):1094-1098.

4. Guo S, DiPietro LA. Factors affecting wound healing. J Dent Res. 2010; 89(3):219-229.

5. Mustoe TA, O'Shaughnessy K, Kloeters O. Chronic wound pathogenesis and current treatment strategies: A unifying hypothesis. Plast Reconstr Surg. 2006;117(7 Suppl):35S-41S.

6. Wong VW, Crawford JD. Vasculogenic cytokines in wound healing. Biomed Res Int. 2013;2013:190486.

7. Tuttolomondo A, La Placa S, Di Raimondo D, et al. Adiponectin, resistin and IL-6 plasma levels in subjects with diabetic foot and possible correlations with clinical variables and cardiovascular co-morbidity. Cardiovasc Diabetol. 2010;9:50.

8. Iversen MM, Tell GS, Riise T, et al. History of foot ulcer increases mortality among individuals with diabetes: Ten-year follow-up of the NordTrøndelag Health Study, Norway. Diabetes Care. 2009;32(12):2193-2199.

9. Escandon J, Vivas AC, Tang J, Rowland KJ, Kirsner RS. High mortality in patients with chronic wounds. Wound Rep Reg. 2011;19(4):526-528.

10. Zillmer R, Trøstrup H, Karlsmark T, Ifversen P, Agren MS. Duration of wound fluid secretion from chronic venous leg ulcers is critical for interleukin-1a, interleukin-1b, interleukin-8 levels and fibroblast activation. Arch Dermatol Res. 2011;303(8):601-606.

11. Trøstrup H, Bjarnsholt T, Kirketerp-Møller K, Høiby N, Moser C. What is new in the understanding of non-healing wounds epidemiology, pathophysiology, and therapies. Ulcers. 2013;2013:625934.

12. Trøstrup $H$, Lundquist $R$, Christensen LH, et al. S100A8/A9 deficiency in nonhealing venous leg ulcers uncovered by multiplexed antibody microarray profiling. Br J Dermatol. 2011;165(2):292-301.

13. Eming SA, Koch M, Krieger A, et al. Differential proteomic analysis distinguishes tissue repair biomarker signatures in wound exudates obtained from normal healing and chronic wounds. J Proteome Res. 2010;9(9):4758-4766.

14. Panee J. Monocyte chemoattractant protein 1 (MCP-1) in obesity and diabetes. Cytokine. 2012;60(1):1-12.

15. Kim CS, Park HS, Kawada T, et al. Circulating levels of MCP-1 and IL-8 are elevated in human obese subjects and associated with obesityrelated parameters. Int J Obes (Lond). 2006;30(9):1347-1355.

16. Su D, Li Z, Li X, et al. Association between serum interleukin- 6 concentration and mortality in patients with coronary artery disease. Mediators Inflamm. 2013;2013:726178.

17. Souza JR, Oliveira RT, Blotta MH, Coelho OR. Serum levels of interleukin-6 (IL-6), interleukin-18 (IL-18) and C-reactive protein (CRP) in patients with type-2 diabetes and acute coronary syndrome without ST-segment elevation. Arq Bras Cardiol. 2008;90(2):86-90.

18. Apostolakis S, Vogiatzi K, Amanatidou V, Spandidos DA. Interleukin 8 and cardiovascular disease. Cardiovasc Res. 2009;84(3):353-360.

19. Koenig W, Khuseyinova N. Biomarkers of atherosclerotic plaque instability and rupture. Arterioscler Thromb Vasc Biol. 2007;27(1):15-26.

20. Amasyali B, Kose S, Kursaklioglu H, Barcina C, Kilicb A. Monocyte chemoattractant protein-1 in acute coronary syndromes: Complex vicious interaction. Int J Cardiol. 2009;136(3):356-357.

21. Dinh T, Tecilazich F, Kafanas A, et al. Mechanisms involved in the development and healing of diabetic foot ulceration. Diabetes. 2012; 61(11):2937-2947.

22. Levi M, van der Poll T, Büller HR. Bidirectional relation between inflammation and coagulation. Circulation. 2004;109(22):2698-2704.
23. van der Wal AC, Becker AE. Atherosclerotic plaque rupture: Pathologic basis of plaque stability and instability. Cardiovasc Res. 1999;41(2): 334-344.

24. Li A, Dubey S, Varney ML, Dave BJ, Singh RK. IL-8 directly enhanced endothelial cell survival, proliferation, and matrix metalloproteinases production and regulated angiogenesis. J Immunol. 2003;170(6): 3369-3376.

25. Moreau M, Brocheriou I, Petit L, Ninio E, Chapman MJ, Rouis M. Interleukin-8 mediates downregulation of tissue inhibitor of metalloproteinase-1 expression in cholesterol-loaded human macrophages: Relevance to stability of atherosclerotic plaque. Circulation. 1999;99(3): 420-426.

26. Barrientos S, Stojadinovic O, Golinko MS, Brem H, Tomic-Canic M. Growth factors and cytokines in wound healing. Wound Repair Regen. 2008;16(5):585-601.

27. Ait-Oufella $\mathrm{H}$, Taleb S, Mallat Z, Tedgui A. Recent advances on the role of cytokines in atherosclerosis. Arterioscler Thromb Vasc Biol. 2011; 31(5):969-979.

28. Bromley SK, Mempel TR, Luster AD. Orchestrating the orchestrators: Chemokines in control of T cell traffic. Nat Immunol. 2008;9(9):970-980.

29. de Jager SC, Bongaerts BW, Weber M, et al. Chemokines CCL3/MIP1a, CCL5/RANTES and CCL18/PARC are independent risk predictors of short-term mortality in patients with acute coronary syndromes. PLoS One. 2012;7(9):e45804.

30. Robson MC. The role of growth factors in the healing of chronic wounds. Wound Rep Reg. 1997;5(1):512-517.

31. Sapienza P, di Marzo L, Borrelli V, et al. Basic fibroblast growth factor mediates carotid plaque instability through metalloproteinase-2 and -9 expression. Eur J Vasc Endovasc Surg. 2004;28:89-97.

32. Petreaca ML, Yao M, Ware C, Martins-Green MM. Vascular endothelial growth factor promotes macrophage apoptosis through stimulation of tumor necrosis factor superfamily member 14 (TNFSF14/ LIGHT). Wound Repair Regen. 2008;16(5):602-614.

33. Steffel J, Lüscher TF, Tanner FC. Tissue factor in cardiovascular diseases: Molecular mechanisms and clinical implications. Circulation. 2006;113(5):722-731.

34. Morceau F, Dicato M, Diederic M. Pro-inflammatory cytokine-mediated anemia: Regarding molecular mechanisms of erythropoiesis. Mediators Inflamm. 2009;2009:405016.

35. Salmon-Ehr V, Ramont L, Godeau G, et al. Implication of interleukin-4 in wound healing. Lab Invest. 2000;80(8):1337-1343.

36. Dehne N, Tausendschön M, Essler S, Geis T, Schmid T, Brüne B. IL-4 reduces the proangiogenic capacity of macrophages by down-regulating HIF-1a translation. J Leukoc Biol. 2014;95(1):129-137.

37. Ahdieh $M$, Vandenbos T, Youakim A. Lung epithelial barrier function and wound healing are decreased by IL- 4 and IL- 13 and enhanced by IFN-g. Am J Physiol Cell Physiol. 2001;281(6):C2029-2038.

38. Cianfarani F, Tommasi R, Failla CM, et al. Granulocyte/macrophage colony-stimulating factor treatment of human chronic ulcers promotes angiogenesis associated with de novo vascular endothelial growth factor transcription in the ulcer bed. Br J Dermatol. 2006; 154(1):34-41.

39. Dogra S, Sarangal R. Summary of recommendations for leg ulcers. Indian Dermatol Online J. 2014;5(3):400-407.

40. Beidler SK, Douillet CD, Berndt DF, Keagy BA, Rich PB, Marston WA. Inflammatory cytokine levels in chronic venous insufficiency ulcer tissue before and after compression therapy. J Vasc Surg. 2009;49(4): 1013-1020. 